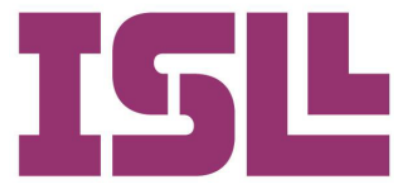

Número 8.

Julio de 2017

\section{Lectura y educación en España: análisis longitudinal de las leyes educativas generales}

\section{Reading and education in Spain: longitudinal analysis of general education laws}

Pág. 79 a la 90

\section{Keywords}

Law, reading, education, habit of reading, libraries

\section{Abstract:}

Reading has emerged as one of the fundamental pillars of education in recent years, gaining a notorious relevance in consideration with other materials. Thus, the reading habit or reading skills are two of the most studied in recent decades and its social projection elements inevitably passes through the general education laws.

In the present study, a comparative analysis of all the laws that emerged in Spain in the era of democracy, a spectrum that occupies nearly fifty years of history of the country, intending to trace how it is treated is made the subject of reading, reading habits and reading skills from the legal basis of education.

For this purpose, a thorough search of the most important to narrow that field and tracking the results in terms of appointments to the chosen terms are analyzed keywords is performed. The findings are compelling because, for example, the keyword "read" not even once appear in any of the texts consulted, nor the term "reading literacy" that although it is more recent, and it was used from the 2000, so that from 2002 to LOCE (although not enter into force because it was not his approach and results evaluated) and the following laws could have been raised. Perhaps if the legal basis from which the rights and duties of society are consolidated argument fails on the field of reading is normal, systematically, the Spanish students rate below average in international tests PISA and PIRLS. 
Este estudio se llevó a cabo gracias a la estancia de investigación de la autora en la Universidad de Alicante, en el departamento de Didáctica de la Lengua y la Literatura, bajo la supervisión del Dr. Díez Mediavilla.

\section{Introducción}

Ya apuntaba Steinbeck que "la tarea de aprender a leer es el mayor esfuerzo que emprende la mente humana" (Díez, 2015, 43).

Eco (2010):

El libro es como la cuchara, el martillo, la rueda, las tijeras. Una vez se ha inventado, no se puede hacer nada mejor. El libro ha superado la prueba del tiempo... Quizás evolucionen sus componentes, quizás sus páginas dejen de ser de papel, pero seguirá siendo lo que es.

La lectura se ha consolidado en la última década como un parámetro fundamental con entidad propia no solo dentro de la educación sino de la sociedad en general. Tanto es así, que se han conformado desde principios del siglo XXI distintas herramientas internacionales (PISA, PIRLS) centradas en la evaluación de la comprensión y la competencia lectoras (Jiménez-Pérez, 2014) ya que los estudios demuestran que a mejor competencia lectora mejor competencias básicas (García-Llamas, Quintanal, 2014, 90), que las mejores actuaciones de alumnos son aquellos que manejan mejor vocabulario debido a su competencia lectora (Cain, Oakhill, 2014, 659) y que "la lectura por placer favorece un aumento en las notas escolares" (Dezcallar, Clariana, Cladelles, Badia y Gotzens, 2014, 114) por lo que se plantea el reto de dicha lectura por placer (Ortega, $2015,132)$ ya que así se puede crear un mejor clima escolar, lo que siempre "afecta positivamente a la actitud de los alumnos" (Krüger, Formichella, 2015, 31) incluso se puede predecir los logros en lectura (Froilan, Oros, 2013, 16). Y, aunque en general los estudiantes perciben que su competencia lectora es buena, sus actuaciones no demuestran dicho nivel (Felipe, Barrios, 2015, 93), cuestión que se puede hacer extensiva a la sociedad española en vista de los resultados en competencia lectora que realiza PISA para los adultos. A pesar de que no hay que olvidar que es necesario entrenar la lectura desde otras perspectivas, por ejemplo, la lectura extensiva (Milliner, Cote, 2015, 404), para evitar que las tecnologías de la información y de la comunicación, donde prima la imagen sobre la palabra y la cultura de la inmediatez, y se generan textos cada vez más breves, acostumbren al cerebro a desconectar cada pocas palabras; o que tarea del docente es entrenar al alumnado en el desarrollo de este tipo de habilidades (Milliner, Cote, 2015, 408), se han de tener en cuenta otras variables, como que la lectura está asociada a las emociones (Dávalos, León, 2013, 118). Porque entender que la lectura puede ser otra forma de entender la sociedad ya ayudaría no solo a la mejora la lectura con el fin de comprender sino también para mejorar el conocimiento de la realidad (Swanson et al., 2015, 2).

Surge la pregunta de que si los universitarios, esos que están estudiando para desempeñar en un futuro su carrera profesional como docentes, muestran actuaciones en competencia lectora peores de las que piensan, sobre todo en pruebas de textos expositivos y argumentativos (Felipe, Barrios, 2015, 93) están adecuadamente preparados para afrontar su futura labor docente.

ISL, vol. 8, 2017, págs. 79-90 ISSN: $2340-8685$
Jiménez Pérez, E. (2017): Lectura y educación en España: análisis longitudinal de las leyes educativas generales, Investigaciones Sobre Lectura, 8, 79-90. 
Tarea de las familias educar en valores, incluyendo los culturales en los que tenga cabida el desarrollo del hábito lector, como tarea de las instituciones gubernamentales es legislar desde la base general de las leyes de la educación sobre el papel que la lectura ha de tener no ya en las aulas sino en la sociedad. Los planes de fomento de la lectura, no recogidos en las leyes generales de España, se han afianzado en conjunción con la mejora del uso de las bibliotecas escolares, y las instituciones públicas dedican partidas económicas exclusivamente para este ámbito (premios de animación a la lectura María Moliner, Plan Nacional al Fomento de la lectura, MECD; premios Líber, FGE...), aunque habitualmente a nivel regional (Plan de Lectura del País Vasco, Eusko Jaurlaritza; Plan de fomento de la lectura, Murcia...). Incluso se establece socialmente la "doble conciencia" (Edwards, Turner, 2011, 637) de si el hábito ha de fomentarse en las aulas o en el seno familiar, siendo conscientes de que no existe una costumbre mediática de poner en valor la lectura, como también ocurre en otros países como Portugal (García-Delgado, 2016, 18).

Pero la realidad legal en cuanto a la ley general de educación, entendida como la propuesta base del gobierno central, parece caminar paralela a este, ya no tan nuevo, escenario en la enseñanza de nuestro país, varios pasos por detrás y sin opción a confluir aparentemente. Como explica Xavier Melgarejo en su conferencia CICLIP, en Finlandia la lectura forma parte de la sociedad y familias enteras acuden quincenalmente a la biblioteca, incluso con maletas para llenarlas de libros, cuestión alejada de la realidad bibliotecaria en España, a pesar de la calidad de las mismas. Una de las causas, según Melgarejo apunta, es el prestigio social del docente, auspiciado por unas leyes de educación flexibles pero en los que la lectura está presente.

Así, con el presente estudio, se realiza un análisis comparativo de las distintas leyes surgidas a lo largo de los últimos cincuenta años de historia de la educación en España con la intención de analizar hasta qué punto es relevante el tema de la lectura en ellas. Se parte de las ocho leyes generales de educación publicadas en BOE a partir de 1970 como eje vertebrador de la comparativa: la LGE (1970), LOECE (1980), LODE (1985), LOGSE (1990), LOPEG (1995), LOCE (2002), LOE (2006) y LOMCE (2013).

Se utiliza una serie de palabras clave para buscar en los textos legales verificándose si cada una de ellas hace referencia a la lectura y no a otro ámbito, además de poder referirse al uso de las bibliotecas, de un plan de fomento para leer o del papel de las TIC como instrumento de respaldo. A saber: leer, lectura, lector, comprensión (lectora), competencia (lectora), lectora, hábito (lector), biblioteca, tecnología (Tecnología de la Información y la Comunicación).

Se espera que ninguna ley o casi ninguna recoja le lectura entre sus prioridades educativas o la regule aunque sea en un segundo plano, entendiéndose una relación directa en primera instancia entre estas leyes generales y su implicación en el papel que la lectura 
viene desarrollando en la sociedad española en la actualidad. Así, se parte de la hipótesis de que el papel de la lectura no ha sido relevante en dichas leyes puesto que no ha tenido repercusión social hasta ahora y que dicho protagonismo se ha dejado exclusivamente en manos de la concreción legal autonómica en su caso, además de que su origen ha sido bastante tardío con respecto a la génesis de las mencionadas leyes.

\section{Método}

\subsection{Muestra}

Para el presente estudio se ha recopilado la información divulgada por el gobierno en sus respectivas leyes orgánicas publicadas en el BOE a lo largo de los últimos cincuenta años de historia que constituyen el periodo democrático actual y fin de la etapa franquista.

\subsection{Instrumentos}

\section{La LGE, 1970}

Ley General de Educación y Financiación de la Reforma Educativa, ley 14/1970, vigente hasta 1980, reguló por primera vez en ese siglo todo el sistema educativo español para responder al cambio social y económico de aquella época. Así, se estructura el sistema en cuatro niveles: Preescolar, Educación General Básica, Enseñanzas Medias y Enseñanza Universitaria, estableció la enseñanza obligatoria hasta los 14 años. Tras ocho años de EGB, Educación General Básica, en un sistema no discriminatorio de escolarización plena, se accedía a BUP Bachillerato Unificado Polivalente. Se trató de establecer una configuración del sistema educativo de forma centralizada, que trajo consigo una uniformidad en la enseñanza.

\section{La LOECE, 1980 (no entró en vigor)}

Ley Orgánica por la que se regula el Estatuto de Centros Escolares (LOECE), con la que se realizó el primer intento normativo de ajustar los principios de la actividad educativa, la organización de los centros docentes y los derechos y deberes de los alumnos a los principios que marcaba la recién aprobada Constitución. El PSOE la recurrió por no respetar las normas fundamentales de la Constitución de 1978 según su criterio, por lo que nunca llegó a entrar en vigor ya que este partido ganó las elecciones en 1982.

\section{La LODE, 1985}

Se refiere a Ley Orgánica del Derecho a la Educación, cuyo objetivo era garantizar para todos el derecho a la educación. Desde esta Ley se intentó garantizar y desarrollar el derecho a la libertad de enseñanza de acuerdo con la Constitución; su novedad más significativa fue que se aprobó la existencia de una doble red de puestos escolares, públicos y privados, estableciéndose un régimen de conciertos al que se pueden acoger centros de titularidad privada para ser financiados con fondos públicos. Esta ley distinguía así entre centros cuyo titular era un poder público o centros privados, que funcionan en 
régimen de mercado, y centros de titularidad privada sostenidos con fondos públicos denominados centros concertados. Además, promocionó la participación en el proceso educativo de todos los sectores implicados quedando a su vez reconocida la libertad de asociación, federación y confederación de los padres y de los propios alumnos.

\section{La LOGSE, 1990}

Ley Orgánica de Ordenación General del Sistema Educativo, regulaba la estructura y organización del sistema educativo en sus niveles no universitarios. En ella se reiteraban los principios y derechos reconocidos en la Constitución y en la LODE. Encaminada a conseguir objetivos como la regulación efectiva de la etapa previa a la escolaridad obligatoria, la reforma profunda de la formación profesional, y la conexión entre las Enseñanzas de Régimen General y Especial, la nueva estructura del sistema educativo establecía en su régimen general las etapas de Educación Infantil, Educación primaria, Educación Secundaria (que comprenderá la Educación Secundaria Obligatoria, el Bachillerato y la Formación Profesional de grado medio), Formación Profesional de grado superior, y Educación Universitaria. Sus reformas más significativas consistieron en derogar definitivamente la LGE de 1970 y, por otro lado, estableció la escolaridad obligatoria hasta los 16 años. También atribuyó competencias a las Comunidades Autónomas de manera muy significativa. Asimismo, se introdujo el concepto de "necesidades educativas especiales" y dedicó una parte importante de su redacción a la educación de personas adultas.

\section{La LOPEG, 1995}

Es la Ley Orgánica de Participación, Evaluación y Gobierno de los Centros Docentes (LOPEG). Trabajó la adecuación entre el sistema y la demanda social, valorándose aspectos como la evaluación de los centros, de la función pública docente, de la labor directiva y de la inspección, así como de la formación del profesorado. Se desarrollaron las funciones, condiciones de acceso y ejercicio de la inspección educativa estableciéndose así los aspectos que las distintas Comunidades Autónomas habían de desarrollar, entre otros, las condiciones para garantizar la escolarización del alumnado con necesidades educativas especiales en centros públicos, los criterios de admisión de alumnos en algunas enseñanzas, los convenios con centros de Formación Profesional o de programas de Garantía Social y los planes de formación de profesorado.

\section{La LOCE, 2002 (no entró en vigor)}

La Ley Orgánica de Calidad de la Educación (LOCE) fue la reforma educativa propugnada por el Partido Popular pero nunca llegó a aplicarse porque ganó las elecciones el PSOE.

Modificaba tanto la Ley Orgánica Reguladora del Derecho a la Educación (LODE) de 1985, como la Ley Orgánica de Ordenación General del Sistema Educativo (LOGSE) de 1990, y la Ley Orgánica de la Participación, la Evaluación y el Gobierno de 
los Centros Docentes (LOPEG) de 1995. Esta ley, la LOCE, proponía una serie de medidas con el principal objetivo de lograr una educación de calidad para todos.

\section{La LOE, 2006}

La Ley Orgánica de la Educación (LOE) contó con un gran consenso para su aprobación. Introdujo la novedad de la asignatura de Educación para la Ciudadanía y los Derechos Humanos y, a su vez, declaró la asignatura de Religión como voluntaria, ya que hasta ahora había sido obligatoria. Esta ley y su estructuración de la nueva asignatura supusieron un perjuicio para Lengua y Literatura ya que se redujeron las horas de su impartición. Por otro lado, los alumnos podían pasar de curso teniendo asignaturas suspensas, así que estuvo en entredicho por profesorado y familias.

\section{La LOMCE. 2013}

La Ley Orgánica para la Mejora de la Calidad Educativa (LOMCE) reforma la LOE 2006 y la LOGSE 1990. Esta ley es la que actualmente está en vigor y se presenta cargada de animadversión por parte de multitud de sectores educativos: profesores, alumnos, partidos políticos... (huelga general que se produjo del 16 de mayo de 2013). Los principales objetivos que persigue esta Ley Orgánica son reducir la tasa de abandono temprano de la educación, mejorar los resultados educativos de acuerdo con criterios internacionales (tanto en la tasa comparativa de alumnos y alumnas excelentes, como en la de titulados de Educación Secundaria Obligatoria), mejorar la empleabilidad y estimular el espíritu emprendedor de los estudiantes. Los principios sobre los cuales impera la reforma son, fundamentalmente, el aumento de la autonomía de los centros, el esfuerzo de la capacidad de gestión de la dirección de centros, las evaluaciones externas de fin de etapa, la racionalización de la oferta educativa y la flexibilización de las trayectorias. Junto a estos principios es necesario destacar tres ámbitos sobre los que la LOMCE hace especial incidencia con vistas a la transformación del sistema educativo: las Tecnologías de la Información y la Comunicación, el fomento del plurilingüismo, y la modernización de la Formación Profesional española.

\subsection{Procedimiento}

Para la confección de la tabla comparativa, se han tenido en cuenta las ocho leyes de la democracia y fin de la dictadura independientemente de si llegaron a entrar o no en vigor que se describen de forma general en el apartado instrumentos. No se ha sometido a evaluación la posible repercusión de sus actuaciones sino la contemplación en sus redacciones de ciertas palabras clave, así pues, por ese motivo, no se han descartado las leyes que no llegaron a entrar en vigor por uno u otro motivo ya que de lo que se trata es de analizar la perspectiva, lectora o no, de su redacción.

ISL, vol. 8, 2017, págs. 79-90 ISSN: $2340-8685$
Jiménez Pérez, E. (2017): Lectura y educación en España: análisis longitudinal de las leyes educativas generales, Investigaciones Sobre Lectura, 8, 79-90. 


\begin{tabular}{llll}
\hline \multicolumn{1}{c}{ Ley 1 } & \multicolumn{1}{c}{ Ley $\mathbf{2}$} & \multicolumn{1}{c}{ Ley $\mathbf{3}$} & \multicolumn{1}{c}{ Ley $\mathbf{4}$} \\
\hline LGE 1970 & LOECE 1980 & LODE 1985 & LOGSE 1990 \\
Ley 5 & Ley 6 & Ley 7 & Ley 8 \\
LOPEG 1995 & LOCE 2002 & LOE 2006 & LOMCE 2013 \\
\hline
\end{tabular}

Tabla 1. Leyes educativas en democracia. Fuente: elaboración propia

Una vez localizados los documentos a través de internet, consultando los BOE, se procede a su análisis mediante la herramienta buscar del procesador de texto para formato PDF.

Las palabras clave utilizadas han sido las siguientes: leer, lectora, lector, comprensión lectora, competencia lectora, hábito lector, biblioteca o tecnología de la información y la comunicación (TIC), siguiendo la categorización que seguidamente se detalla:

1. Leer

2. Lectora

3. Lector

4. Comprensión lectora

5. Competencia lectora

6. Hábito lector

7. Biblioteca

8. TIC

9. Lectura

\begin{tabular}{|c|c|c|c|c|c|c|c|c|c|}
\hline \multirow{2}{*}{\multicolumn{10}{|c|}{ Biblioteca }} \\
\hline & & & & & & & & & \\
\hline \multicolumn{10}{|l|}{ LOECE } \\
\hline \multicolumn{10}{|l|}{ LODE } \\
\hline \multicolumn{10}{|l|}{ LOGSE } \\
\hline \multicolumn{10}{|l|}{ LOPEG } \\
\hline LOCE & & & & & & $\mathrm{X}$ & $\mathrm{X}$ & & \\
\hline LOE & & $\mathrm{X}$ & & $\mathrm{X}$ & & $\mathrm{X}$ & $\mathrm{X}$ & & \\
\hline LOMCE & & $\mathrm{X}$ & & $\mathrm{X}$ & & & & & \\
\hline
\end{tabular}

Tabla II. Comparación de palabras clave y leyes educativas Fuente: elaboración propia

En primer lugar, se ha seleccionado este conjunto de palabras clave para ejercer una búsqueda acotada del concepto de lectura. Leer se ha establecido como palabra clave fundamental sobre la que pivotan el resto de los conceptos. Los términos lectora y lector no refieren el mismo concepto diferenciando sexo, sino que responde a la necesidad de diferenciar entre lector, entendido como individuo que ejerce la actividad de leer, y lectora, intentando abarcar toda actividad o situación que requiera de este adjetivo.

Además, se establece una diferenciación entre comprensión y competencia lectora (Jiménez, 2014) para analizar si alguna de las leyes responde a los parámetros PISA en la nomenclatura de comprensión y competencia referidos a la lectura, informes 
internacionales en los que se pasó de nombrar la comprensión lectora (PISA 2000) a competencia lectora (PISA 2003), en su traducción al español.

Se ha añadido el concepto de hábito lector en función de la relación que pudiera establecerse entre ley sobre lectura y parámetros tales como ámbito de aplicación, nivel de concreción, horas de dedicación para crearlo, métodos para fomentarlo, recursos para fomentarlo, etc.

Con respecto a la palabra clave "biblioteca", se ha elegido para delimitar la posible existencia de una acción determinada por parte de alguna de las leyes en relación a la lectura en su nivel de concreción.

Por su parte, la última palabra clave, tecnología de la información y la comunicación (TIC), da un paso cualitativo al frente en la búsqueda de recursos acordes con la realidad educativa en la que ya lleva inmerso varios años el sistema de educación español.

\subsection{Datos}

El barrido de las leyes a partir de las palabras clave da como resultado que el término "leer", primera de estas claves, no aparece en ninguna de las ocho leyes señaladas.

Atendiendo a la segunda palabra clave, "lectora", entendida como adjetivo que califica a los sustantivos "competencia" o "comprensión", solo aparecen en las dos últimas leyes mencionadas: la LOE de 2006 y la LOMCE de 2013.

De igual forma, el término "lector" tampoco aparece en ninguna de ellas, como también ocurría con "leer".

Así, el término "competencia (lectora)", acuñado por PISA a principios del 2000, no aparece en ninguna de las leyes, incluyendo las más actuales como la LOMCE. Sin embargo, "comprensión (lectora)" queda registrado a partir de la LOE (2006) y se repite en la última ley a la que precede, la LOMCE (2013), quizás por desconocer la tendencia surgida a principios de siglo de nominar como competencia lectora a la antigua comprensión (Jiménez, 2014).

Con respecto a la palabra clave "hábito" se han encontrado coincidencias en las tres últimas leyes existentes, LOCE, LOE, LOMCE, que, aunque se esperaba acompañara a otra de las palabras clave, lector, aparece registrada junto al complemento del nombre "de lectura" en el caso de la LOCE y la LOE, mientras que en la LOMCE no hace 
referencia al ámbito lector sino que señala directamente a la convivencia, de trabajo, o la relación entre los propios discentes.

Llegado el turno de la palabra clave "biblioteca" destacar que en la LGE (1970), LOECE (1980), LODE (1985), la LOGSE (1990) y la LOPEG (1995) no registran ninguna entrada y es solo a partir de la LOCE (2002) cuando por fin aparece por primera vez el término para continuar en sus leyes posteriores excepto la última, la LOMCE (2013).

Por último, la palabra clave "tecnología", con la que se ha querido cercar las Tecnologías de la Información y la Comunicación (TIC) en relación con la lectura, el acto de leer o la comprensión y competencia lectoras, no aparece en ninguna de las leyes, incluyendo la LOMCE (2013). Sin embargo, sí aparece el término Nuevas Tecnologías en esta última, además de en otras como la LOCE (2002), aunque la expresión quede obsoleta por la realidad constatada de que hace tiempo que ya no son nuevas. Sin embargo, el término tecnología per se ya aparece en la LOGSE (1990) en referencia a los tipos de bachillerato y se concreta en una asignatura. Solo en la LOE (2006) aparece el término correctamente enunciado como TIC.

Es decir, de las 64 opciones de que apareciera alguna palabra clave en relación con la lectura solo se han registrado 8 , esto es, el $87.5 \%$ de las posibilidades esfumadas

\section{Conclusiones}

De que la lectura es un pilar fundamental no solo de la educación sino de todo el contexto social, más allá de lo meramente cultural, no cabe duda. Leer mejora adelanta hasta en un curso a los niños con hábito según la OCDE al igual que reduce los índices de fracaso escolar, por lo tanto, es de vital importancia que se vertebre desde las leyes fundamentales de educación las actuaciones pertinentes en este campo que, con posterioridad, quedarán concretadas según las autonomías.

Las ocho leyes generales educativas de la democracia y fin de la dictadura se plantean como columna vertebral del sistema de educación de España, por lo que estudiar qué papel tiene la lectura en ellas se proyecta como una necesidad básica en la tarea de conocer la relación entre el hábito lector y la educación y su proyección en la sociedad actual. El impulso que necesita la lectura pasa inequívocamente por establecerla con categoría estatal básica en el sistema de educación desde una perspectiva amplia, donde deben incluirse desde las bibliotecas, las tecnologías de la información y la comunicación, con especial hincapié en los medios, los profesores, las editoriales y hasta las familias, leer es una cuestión de cultura general y el hábito lector es la forma de mostrar cómo ha calado en la sociedad. 
En resumen, se puede destacar lo significativo de que la palabra "leer" no aparezca en ninguna de las ocho leyes reseñadas. Si ninguna ley recoge el acto lector, leer, que ni siquiera es aludido transversalmente, se está restando la importancia que realmente tiene la lectura y es contraproducente exigir que los niños creen o consoliden un hábito lector si en las leyes generales de educación no solo no se regula, sino que ni se menciona. También es interesante el dato de que el órgano de gobierno educativo de más alto rango de este país no nomine la competencia lectora aun en la reciente ley educativa (LOMCE, 2013) o que en la misma se denomine nuevas tecnologías a las TIC, cuando ya no lo son, o se obvie el tratamiento de las bibliotecas como punto clave en la socialización de la lectura y herramienta primordial para las familias.

Además, se confirma la hipótesis de que era de esperar que en las leyes generales de educación no se realizara mención, alusión al acto de leer o se legislara en el ámbito de la lectura ya que las leyes educativas trascienden en la sociedad y la lectura no es prioridad cultural en este país. De hecho, rara vez aparecen noticias en TV, el medio de comunicación tradicional con más seguidores, relacionadas con la lectura, aunque sí con el fútbol ya que socialmente se considera a efectos prácticos una manifestación cultural infinitamente más importante que leer.

Cabe destacar que a pesar de esta forma de ignorar la lectura de forma general en las leyes nacionales de educación, el presente estudio no incluye un análisis de la concreción autonómica de dichas leyes por lo que este trabajo abre las puertas para realizar la misma mecánica analítica en un nivel inferior, el autonómico, ya que es cierto que dichos gobiernos sí legislan en el terreno educativo sobre la lectura (por ejemplo, Murcia establece un horario lector que se materializa en media hora al día o Andalucía en una hora diaria), así, se podría establecer la relación entre sus leyes y la repercusión social en su territorio.

Sin embargo, este estudio, sin precedentes en el campo de la investigación educativa, pone de manifiesto la necesidad de un cambio de perspectiva en las leyes básicas de educación generadas por el Ministerio ya que la lectura, no entendida como parte de la asignatura de lengua, está demostrado que incide de forma significativa en el amplio espectro educativo que todos los alumnos deben afrontar a lo largo de su vida y, yendo aún más allá, en la percepción objetiva de la realidad que le rodea ya que el acto de la comunicación, también por escrito, es lo que hace de ser humano un ser social.

Pero no solo desde el punto de vista de las instituciones gubernamentales depende la mejora de este aspecto de la sociedad, que implica con especial interés al alumnado español de todas las edades, también de todos los actores sociales implicados directamente en la consolidación del acto lector como una parte de la idiosincrasia cultural del país: docentes (que en muchos casos no son lectores y que, por extensión, no podrán 
transmitir esa pasión al carecer de ella), familias (demasiado preocupadas en conciliar vida familiar y laboral, y más partidarias de cultivar otras formas de distracción que no sea la lectura), instituciones, editoriales (que ante la posibilidad de invertir en hacer su imagen más visible y dar importancia publicitaria a los eventos que patrocinan prefieren seguir la inercia de lo ya implantado) y medios de comunicación (que deberían dedicar el mismo tiempo de emisión a noticias de fútbol que de lectura, por ejemplo).

Quizás, haciendo especial hincapié en el punto de partida legal, y en la necesidad de llegar a un pacto por la educación en la que se contemple la regularización del tratamiento de la lectura a todos los niveles educativos, se pueda empezar a cultivar un campo que aún al día de hoy no da los frutos que se esperan.

\section{Referencias bibliográficas}

Cain, K., Oakhill, J. (2014). Reading comprehension and vocabulary: is vocabulary more important for some aspects of comprehension? L'année psycologique/Topics in Cognitive Psycology, 114, 647-662.

Dezcallar, T.; Clariana, M.; Cladelles, R. Badia, M. y Gotzens, C. (2014). La lectura por placer: su incidencia en el rendimiento académico, las horas de televisión y las horas de videojuegos. Ocnos, 12, 107-116.

Diez, A. García, A. (2015). La evaluación de la competencia lectora. Lenguaje y textos, $41,35-44$.

Eco, U. (2010). Nadie acabará con los libros. Barcelona: RHM.

Edwards, P. A., Turner, J. D. (2011). Family Literacy and Reading Comprehension en Felipe, A., Barrios, E. (2015). Prospective teachers' reading competence: perceptions and performance in a reading test. 15th International Conference SEDLL, Valencia, 87-93.

Froilan, J. M., Oros, E. (2013). Intrinsic Motivation, Perceived Competence and Classroom Engagement as Longitudinal Predictors of Adolescent Reading Achievement, Educational Psychology, 34, 119-132.

García-Delgado, B. (2016). Análisis de las noticias en Portugal: Jornal de noticias 2012. ISL, 6, 7-24.

García-Llamas, J. L. y Quintanal, J. (2014). El desarrollo lector y su relación con la mejora de las competencias básicas. Ocnos, 11, 71-91.

Israel, S. E, Duffy, G. G. (edits.) Handbook of Research on Reading Comprehension, London, UK: Routledge, 622-641. 
Jiménez-Pérez, E. (2014). Comprensión lectora Vs competencia lectora: qué son y qué relación existe entre ellas. ISL, 1, 65-74.

Krüger, N., Formichella, M. M. (2015). Más allá de los logros cognitivos: la actitud hacia la escuela y sus determinantes en España según PISA 2008. Revista de Educación, 367, 10-35.

Milliner, B., Cote, T. (2015). One year of extensive reading on mobile devices: engagement and impressions. In F. Helm, L. Bradley, M. Guarda, \& S. Thouësny (Eds), Critical CALL - Proceedings of the 2015 EUROCALL Conference, Padova, Italy, 404-409

Ortega, F. J. (2015). La importancia de la lectura y las tecnologías en el aprendizaje del español para inmigrantes. ISL, 3, 123-133

Swanson, E., Wanzek, J., Vaughn, S., Roberts, G., Fall, A. M. (2015). Improving reading comprehension and social studies knowledge among middle school students with disabilities. SAGE, 1-17.

VV.AA. (1970). LGE, BOE 187, 12525-12546.

VV.AA. (1980). LOECE, BOE 154, 14633-14636.

VV.AA. (1985). LODE, BOE 159, 21015-21022.

VV.AA. (1990). LOGSE, BOE 238, 28927-28942.

VV.AA. (1995). LOPEG, BOE 278, 33651-33665.

VV.AA. (2002). LOCE, BOE 307, 45188-45220.

VV.AA. (2006). LOE, BOE 106, 1-107.

VV.AA. (2013). LOMCE, BOE 295, 97858-97921.

ISL, vol. 8, 2017, págs. 79-90 ISSN: 2340-8685
Jiménez Pérez, E. (2017): Lectura y educación en España: análisis longitudinal de las leyes educativas generales, Investigaciones Sobre Lectura, 8, 79-90. 